\title{
Double Band Double-Pulley Repair for Articular Surface Partial Tears of the Supraspinatus in Throwing Athletes: Improving the Transtendon Technique
}

\author{
Osvaldo Garcia Martinez ${ }^{1}$, Eduard Buess ${ }^{2}$, Diosveny Gonzales Hernandez ${ }^{1}$ \\ ${ }^{1}$ Orthopaedic Department, Hospital Provincial Docente, Ciego de Avila, Cuba; ${ }^{2}$ Sonnnenhof Clinic, Shouldercare Institute, Berne, \\ Switzerland. \\ Email: osvaldo_garciamartinez@yahoo.com
}

Received October $9^{\text {th }}, 2012$; revised November $9^{\text {th }}, 2012$; accepted November $16^{\text {th }}, 2012$

\begin{abstract}
Disabled shoulders of throwing athletes typically present with extended undersurface partial tears of the rotator cuff, which include the posterior supraspinatus and the anterior infraspinatus tendon to a variable extent. We propose a modified transtendon repair technique to adequately treat this subset of patients. The repair includes two double-loaded anchors, at the anterior and the posterior end of the tear, respectively. With the help of an angulated penetrator we create a medial and a lateral band of sutures on top of the cuff, producing a broad contact in the tendon-to-bone interface. All the 9 so far operated patients were young men, 7 of them base-ball pitchers, and 5 active in competitive sports. The Constant Score rose from 72 points preoperatively to 99 points at 12 months follow-up. Three of the still active pitchers were able to return to their previous level in sports after one year. The improved footprint contact of our novel repair construct should allow for better healing and, therefore, a higher chance of return to competition.
\end{abstract}

Keywords: Partial Tear; Supraspinatus Tendon; Throwing Athlete; PASTA-Lesion; PAINT-Lesion

\section{Introduction}

Partial-thickness rotator cuff tears are a cause of pain and loss of function in the concerned patients [1]. In overhead sports and especially throwing athletes they produce diminution of the performance and incapacity to play [2]. Partial-thickness tears can be on the articular surface, on the bursal side of the tendon, or intratendinous. We are especially interested in tears of the articular side coined PASTA lesions ("partial articular supraspinatus tendon avulsion") by Snyder. In patients of the average and older population, the lesions are typically seen in the anterior supraspinatus tendon. In throwing athletes, however, we observe more extended tears affecting the posterior half of the supraspinatus and the anterior portion of the infraspinatus [2,3]. Their cause seems to be at least partially-internal impingement, as occurring in the extreme cocking phase of the throwing motion sequence. For some of these lesions, characterized by an intratendinous delamination into the infraspinatus, the acronym PAINT ("partial articular tear with intratendinous extension") was introduced by Conway. This pathology that can best be detected on MR arthrography in the abducted and externally rotated (ABER) position of the shoulder [4].

Arthroscopic debridement with or without subacromial decompression [1] of articular surface partial lesions often did not produce satisfying and stable results over time [5]. Increasingly, the need for direct repair was recognized in the literature, because of a high probability of progression into complete tears of up to $35 \%$ after debridement alone [5]. This seems to be true at least for tears involving more than $50 \%$ of the tendons's thickness.

Regarding tear size and location the literature is not precise [5]. We therefore intentionally base our considerations on two different arthroscopic classification systems. First we use a modification of Snyder's classification [1] to grade the antero-posterior extent (width) of the lesion: Grade 1 for tears of less than $1 \mathrm{~cm}$, grade 2 for tears of $1-2 \mathrm{~cm}$, grade 3 of $2-3 \mathrm{~cm}$ and grade 4 for extended, complex tears larger than $4 \mathrm{~cm}$. To describe the medio-lateral extension (depth) of the tear we secondly use the well established Ellman classification with a grade I for tears of $3 \mathrm{~mm}$, grade II for tears of $3-6 \mathrm{~mm}$ and grade III for tears deeper than $6 \mathrm{~mm}$ equivalent to more than half of the tendon thickness.

Extended partial-thickness tears of the articular side are regularly seen in our center, where we frequently attend throwing athletes of all levels. This is due to the fact, that baseball is the number one national sports and 
that very few centers practice shoulder arthroscopy at this time. The purpose of this study is to suggest a technical modification for arthroscopic transtendon repair, adequate in obtaining good results in general and improving the chance of the reintegration of active throwing athletes into high level competitive sports.

\section{Operating Technique}

The patient is placed in beach-chair position with a combination of regional and general anesthesia. After a standard diagnostic evaluation of the joint and the subacromial space we place 2 cannulas: a $5 \mathrm{~mm}$ cannula in the posterior standard portal to provide easy repetitive access to the glenohumeral joint and a $8.25 \mathrm{~mm}$ working cannula in the lateral subacromial portal to assist with bursectomy and suture management.

For the visualisation of the subacromial space we prefer a posterolateral viewing portal [6]. Intraarticular debridement with the shaver in the rotator interval will help to delineate the tear from healthy tissue. Tear size regarding its depth and width is measured with a probe after debridement (Figures $\mathbf{1}$ and 2), and a bleeding bone

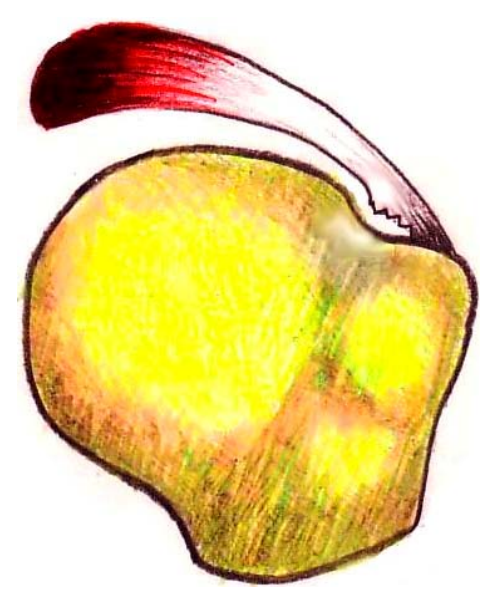

Figure 1. Mediolateral extent of tear size (depth).

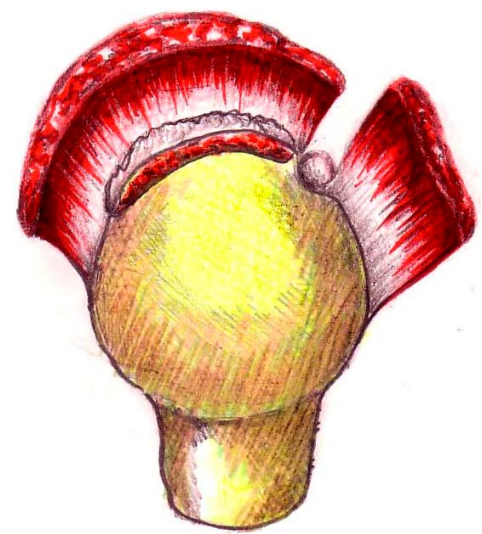

Figure 2. Anteroposterior extent of tear size (width). bed is created on the greater tuberosity. To reach the posterior extension of the tear into the infraspinatus, we like to exchange the instruments placing the arthroscope anteriorly and the shaver posteriorly.

The debrided tear is then repaired to bone with 2 double-loaded titanium anchors: An anterior anchor is placed percutaneously close to the margin of the articular cartilage and at the beginning of the avulsion in the anterior supraspinatus. A second anchor is screwed in through the infraspinatus at the posterior limit of the tear, strictly respecting the bare area. To assist with correct anchor positioning, we place two needles under intraarticular vision. Then we switch to a subacromial view and clean the bursa with the shaver until we can clearly see the needles. By doing so, we later will find the suture limbs without difficulty. The anchor tip should appear inside the torn area at an intermediate position of the mediolateral tear extension. Next we pass the medial suture limbs through healthy tissue about $2-3 \mathrm{~mm}$ medially from the tear border. For easy suture passing we prefer an angulated penetrator and we simultaneously retrieve two same-coloured suture limbs (Figures 3 and $\mathbf{4}$ ).

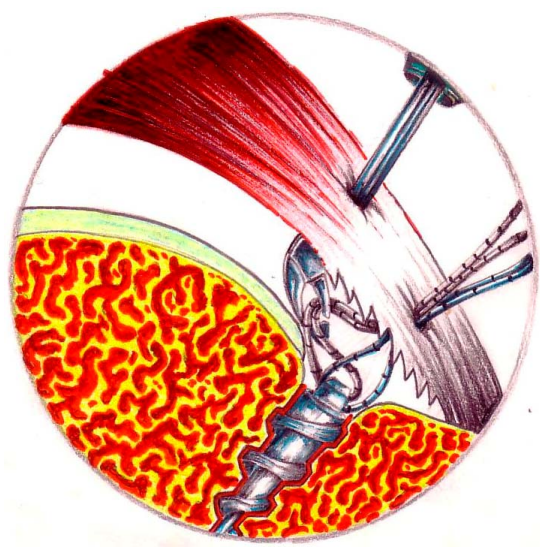

Figure 3. The angulated penetrator retrieves one pair of same-coloured suture limbs through healthy tissue.

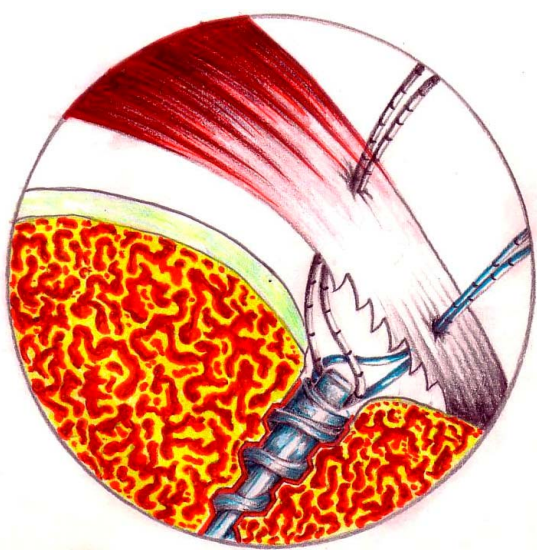

Figure 4. Suture passing completed. 
Once the suture passing completed, we continue by tying a medial band of sutures using the double-pulley principle of Lo and Burkhart [7]. We catch one of the medial suture limbs anteriorly and posteriorly in the subacromial space, deliver them into the lateral working cannula, tie them firmly outside the shoulder and cut the sutures above the knot. We then pull the free ends of these sutures, a maneuver which places the knot an top of the cuff. We then retrieve the two remaining suture limbs and tie them arthroscopically with a static knot, thus completing the medial double-pulley construct. We then proceed in the same fashion with the lateral sutures, creating a second more lateral band of sutures (Figures $\mathbf{5}$ and $\mathbf{6}$ ). That's how we obtain a firm compression of the whole tear area to the debrided bony footprint.

\section{Clinical Experience}

Since 2007 we have peformed a total of 41 PASTA repairs with one or two anchors at our center. The described technique has been used by the first author in 9 patients so far. Mean age of the 9 male patients was 24 years (range 19 - 44). All had a history of throwings

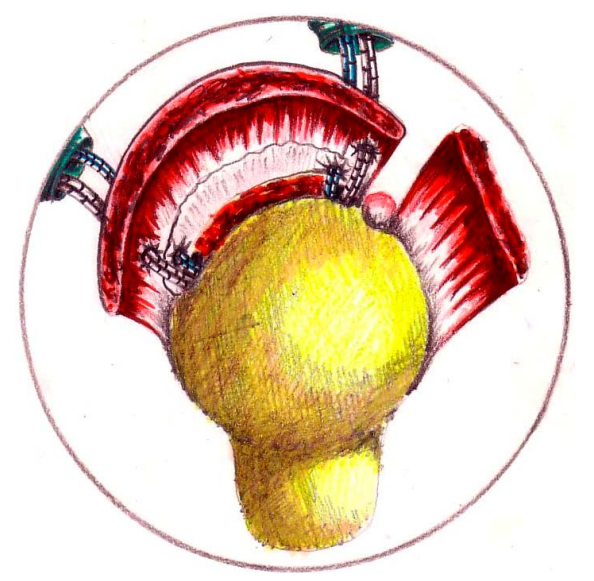

Figure 5. Before knot-tying, articular side view. sports, 7 as professional baseball pitchers, 5 of whom were still active (Table 1). Three patients had SLAP tears (typically associated with PASTA lesions) that were repaired at the same time. The Constant Score rose from 72 points preoperatively to 90 at six and to 99 at 12 months follow-up. No complications were noted. From the 5 active pitchers $3(60 \%)$ could be reintegrated into their teams by starting throwing exercises after 8 months and playing at their previous level after one year.

\section{Discussion}

Traditionally, the repair of high grade undersurface partial-thickness cuff tears consisted in completion and repair with a standard technique for full-thickness tears, meaning that normal tissue had to be sacrificed. In an attempt to avoid this, various authors recommended to spare the healthy tissue on the lateral aspect of the tuberosity and perform a transtendon suture anchor technique [5]. Lo and Burkhart [7] consider that taking down normal tissue accounts for a slower healing and can create a tension mismatch.

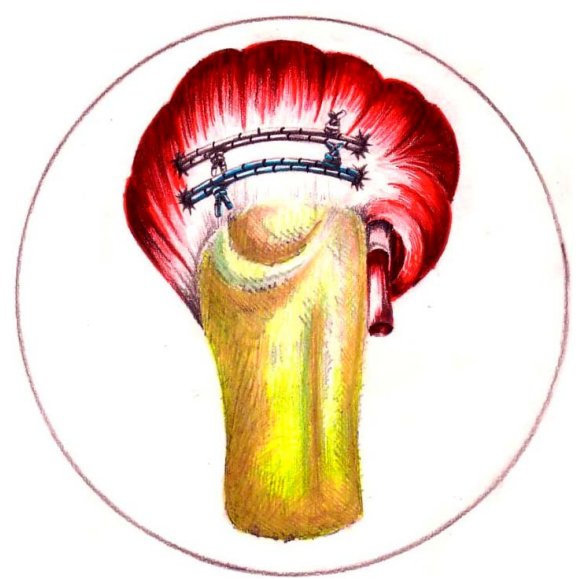

Figure 6. Completed double band double-pulley repair, bursal side view.

Table 1. Patient characteristics and results.

Baseball pitchers (province/national)

Sports

Basketball

Softball

Snyder mod. (width)

Tear Classification

Ellmann (depth)

Constant Score Results
Preop 72 points 7/active 5

1

1

Grade $3(2-3 \mathrm{~cm}) 5$

Grade $4(>3 \mathrm{~cm}) 4$

Grade II (3 - $6 \mathrm{~mm}) 3$

Grade III $(>6 \mathrm{~mm}) 6$

90 points at $6 \mathrm{mt}$

postop

99 points at $12 \mathrm{mt}$ 
Lyons [8] suggested an alternative method using only side-to-side sutures between the supraspinatus and the upper subscapularis. More recently Brockmeyer and colleagues [3] described a technique applying matress sutures without anchors in order to repair the intratendinous component of the lesion. These lesions seem to be typical for throwing atheletes, who according to them, in the past have not shown convincing results with transtendon suture anchor repair. The authors [3] reported promising short term result in 8 patients with their anchorless technique. They caution against non-anatomic fixation of the infraspinatus inside the bare area, which might restrict motion and thus diminish performance in throwing athletes

Although these techniques seem to have had some success, we share the opinion, that a secure fixation of the tendon to bone is a prerequisite for a stable construct, that allows for a favorable biologic healing environment $[6,7]$.

Gonzales-Lomas [9] found in a biomechanical study superior results for a transtendon repair with 2 anchors as compared to tear completion and repair with 4 anchors in a double-row fashion. Peters [10] and colleagues studied the transtendon repair in a sheep model focusing on contact pressure. They also found superiority of the transtendon approach.

We believe that in articular surface partial cuff lesions as seen in younger patients a process of desinsertion of tendon fibres has occurred, that leaves the thickness of the tendon intact and allows for reinsertion with a transtendon suture technique. It is mandatory; however, that superiorly there is good quality tendon tissue (without tendinosis) of at least $25 \%$ of tendon's thickness. The repair should take advantage of the bifocal vascularity coming from both, the bone bed and the bursal side, area of the main blood supply for the cuff. Based on these principles, we prefer for smaller lesions the one anchor technique proposed by Waibl and Buess [6] which uses 3 triangular contact points between the tear and the bone bed, and for more extended tears the double-pulley technique of Lo and Burkhart [7].

We suggest a new double band double-pulley technique for extended partial tears as seen in throwing athletes. The suture position guarantees a broad contact in the tendon-to-bone interface with a potentially improved healing response. Using only one band of sutures, we obtain a repair, in which one tendinous area has no contact with the footprint. In fact, a gap can persist between the medial sutures and the intact lateral tendon fibres (Figure 7). Using two bands, however, we eliminate gap formation (Figure 8). Creating this construct with two large suture bridges, the optimal recreation of the footprint anatomy seems possible. Our technique can help to

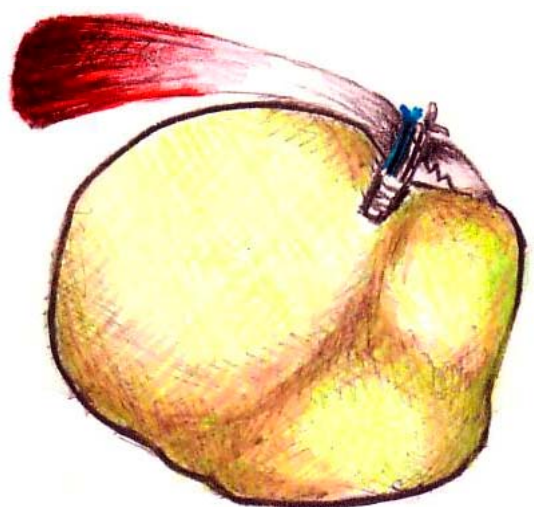

Figure 7. Persisting gap in a single band repair.

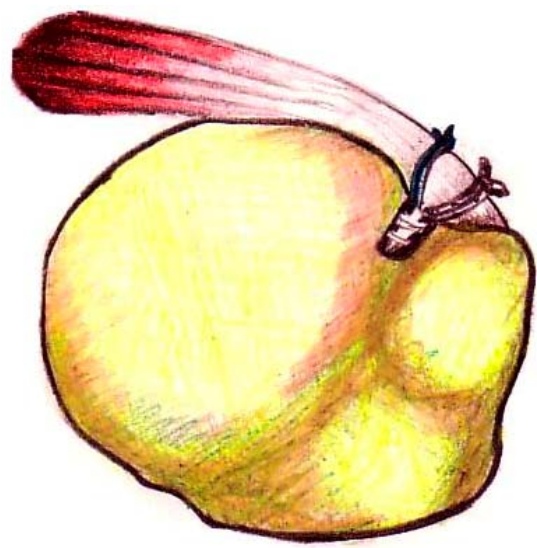

Figure 8. Gap elimination by double band repair.

diminish a possible strangulation effect of the sutures onto the tendon; moreover the medial suture band seems adequate to adress a coexisting PAINT lesion.

The repair of articular surface partial-thickness rotator cuff tears is challenging and bringing back an injured throwing athlete to his previous level remains difficult $[2,3]$. The literature about this subgroup is scarce. We are not aware of biomechanical studies, which focus on the influence of anchors on the throwing synchronism. Maybe one anchor produces an imbalance of the humeral head, but two anchors don't, if placed in an adequate position one anteriorly and one posteriorly.

\section{Conclusion}

We regularly see disabled throwing athletes at our center, due to the fact that baseball is - as in the U.S. - extremely popular all over Cuba. They often present with extended undersurface partial tears of the supra- and infraspinatus, caused most likely by posterosuperior impingement (PSI). These lesions are diffcult to treat. The double band double-pulley transtendon repair has given good results in our patients and shown to be a promising opportunity for high-level throwing athletes. 


\section{Acknowledgements}

The authors would like to thank the artist Liovy Cots Diaz for the artwork.

\section{REFERENCES}

[1] S. J. Snyder, A. F. Pachelli and W. Del Pizzo, "Partial Thickness Rotator Cuff Tears: Results of Arthroscopic Treatment," Arthroscopy: The Journal of Arthroscopic \& Related Surgery, Vol. 7, No. 1, 1991, pp. 1-7. doi:10.1016/0749-8063(91)90070-E

[2] J. E. Conway, "Arthroscopic Repair of Partial-Thickness Rotator Cuff Tears and SLAP Lesions in Professional Baseball Players," The Orthopedic Clinics of North America, Vol. 32, No. 4, 2001, pp. 443-456. doi:10.1016/S0030-5898(05)70213-3

[3] S. F. Brockmeier, C. C. Dodson and S. C. Gamradt, "Arthroscopic Intratendinous Repair of the Delaminated Partial-Thickness Rotator Cuff Tear in Overhead Athletes," Arthroscopy: The Journal of Arthroscopic \& Related Surgery, Vol. 24, No. 8, 2008, pp. 961-965. doi:10.1016/j.arthro.2007.08.016

[4] W. B. Stetson, T. Phillips and A. Deutsch, "The Use of Magnetic Resonance Arthrography to Detect PartialThickness Rotator Cuff Tears," Journal of Bone \& Joint Surgery, Vol. 87, No. 2, 2005, pp. 81-88. doi:10.2106/JBJS.E.00509

[5] E. J. Strauss, M. J. Salata and J. Kercher, "The Arthroscopic Management of Partial-Thickness Rotator Cuff
Tears: A Systematic Review of the Literature," Arthroscopy: The Journal of Arthroscopic \& Related Surgery, Vol. 27, No. 4, 2011, pp. 568-580. doi:10.1016/j.arthro.2010.09.019

[6] B. Waibl and E. Buess, "Partial-Thickness Articular Surface Supraspinatus Tears: A New Transtendon Suture Technique," Arthroscopy: The Journal of Arthroscopic \& Related Surgery, Vol. 21, No. 3, 2005, pp. 376-381. doi:10.1016/j.arthro.2004.11.008

[7] I. K. Lo and S. S. Burkhart, "Transtendon Arthroscopic Repair of Partial-Thickness, Articular Surface Tears of the Rotator Cuff," Arthroscopy: The Journal of Arthroscopic \& Related Surgery, Vol. 20, No. 2, 2004, pp. 214220. doi:10.1053/jars.2001.8017

[8] T. R. Lyons, F. H. Savoie and L. D. Field, "Arthroscopic Repair of Partial-thickness Tears of the Rotator Cuff," Arthroscopy: The Journal of Arthroscopic \& Related Surgery, Vol. 17, No. 2, 2001, pp. 219-223 .

[9] G. Gonzalez-Lomas, M. A. Kippe and G. D. Brown, "In Situ Transtendon Repair Outperforms Tear Completion and Repair for Partial Articular-Sided Supraspinatus Tendon Tears," Journal of Shoulder and Elbow Surgery, Vol. 17, No. 5, 2008, pp. 722-728. doi:10.1016/j.jse.2008.01.148

[10] K. S. Peters, P. H. Lam and G. A. C. Murrell, "Repair of Partial-Thickness Rotator Cuff Tears: A Biomechanical Analysis of Footprint Contact Pressure and Strength in an Ovine Model," Arthroscopy: The Journal of Arthroscopic \& Related Surgery, Vol. 26, No. 7, 2010, pp. 877-884. doi:10.1016/j.arthro.2010.04.007 